Revue de droit comparé du travail et de la sécurité sociale

3 | 2017

Le travail dans l'économie informelle, un défi pour le droit social

\title{
L'informalité en Amérique Latine : l'exemple des politiques de protection sociale des travailleurs informels au Chili
}

Pablo Arellano Ortiz

\section{OpenEdition}

Journals

Édition électronique

URL : https://journals.openedition.org/rdctss/370

DOI : $10.4000 /$ rdctss.370

ISSN : 2262-9815

Éditeur

Centre de droit comparé du travail et de la sécurité sociale

Édition imprimée

Date de publication : 1 septembre 2017

Pagination : 104-115

ISSN : 2117-4350

Référence électronique

Pablo Arellano Ortiz, "L'informalité en Amérique Latine : l'exemple des politiques de protection sociale des travailleurs informels au Chili », Revue de droit comparé du travail et de la sécurité sociale [En ligne], 3 | 2017, mis en ligne le 01 novembre 2018, consulté le 04 décembre 2021. URL : http:// journals.openedition.org/rdctss/370 ; DOI : https://doi.org/10.4000/rdctss.370

\section{(c) $(1)$}

Revue de droit comparé du travail et de la sécurité sociale est mise à disposition selon les termes de la Licence Creative Commons Attribution - Pas d'Utilisation Commerciale - Pas de Modification 4.0 International. 


\section{L'informalité en AmÉriQUe LATINE. L'EXEMPLE DES POLITIQUES DE PROTECTION SOCIALE DES TRAVAILLEURS INFORMELS AU CHILI}

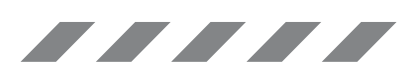

\section{ABSTRACT}

The context of the informal worker is very diverse, and so is manifested in Latin America. The policies adopted to protect informal workers and their families are very diverse, including a variety of actors and institutions. Among these, this paper is concerned with showing as an example those social protection policies implemented in Chile to protect the informal and the poor.

KEYWORDS : Informal worker, Latin America, Chile, social protection, poverty.

\section{RÉSUMÉ}

Le contexte des travailleurs informels est très diversifié, comme on peut le constater en Amérique latine. Les politiques adoptées pour protéger les travailleurs informels et leurs familles sont très variées, et impliquent un grand nombre d'acteurs et d'institutions. Le présent document traite de l'exemple des politiques de protection sociale mises en place au Chili pour protéger les travailleurs informels et les pauvres.

MOTS CLÉS : Travailleurs informels, Amérique latine, Chili, protection sociale, pauvreté. 


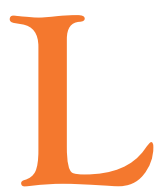

e travail informel connaît plusieurs dénominations ${ }^{01}$, la doctrine parle par exemple du secteur informel, de l'économie informelle ou du secteur non structuré pour désigner le même groupe de personnes exclues de la protection de la loi. Ce concept a été introduit par l'Organisation Internationale du Travail (OIT) ${ }^{02}$, qui souligne que «l'expression " économie informelle " fait référence à toutes les activités économiques de travailleurs et d'unités économiques qui ne sont pas couverts - en vertu de la législation ou de la pratique - par des dispositions formelles. Ces activités n'entrent pas dans le champ d'application de la loi, ce qui signifie que ces travailleurs et ces unités opèrent en marge de la loi : soit ils ne sont pas couverts dans la pratique, ce qui signifie que la loi ne leur est pas appliquée alors même qu'ils opèrent dans le cadre de la loi ; soit encore la loi n'est pas respectée parce qu'elle est inadaptée, contraignante ou qu'elle impose des charges excessives ». L'origine de ce concept pour l'OlT figure dans des travaux qu'elle a réalisés concernant les marchés du travail sur le continent africain, dans les années 1960 et $1970^{\circ 3}$. Aujourd'hui, il est utilisé de manière globale, puisque l'économie informelle est aussi présente dans un grand nombre d'autres régions.

Récemment, l'OIT a donné une définition de l'emploi informel qui est généralement acceptée et comprend les types d'emploi suivants ${ }^{04}$ :

- les travailleurs indépendants possédant leur propre entreprise dans le secteur informel,

- les employeurs possédant leur propre entreprise dans le secteur informel,

- les travailleurs aides familiaux, qu' ils travaillent dans des entreprises formelles ou informelles,

- les membres de coopératives informelles de producteurs,

- les salariés qui ont des emplois informels, employés par des entreprises du secteur formel, par des entreprises du secteur informel ou par des ménages qui les emploient comme travailleurs à domicile salariés, et

- les travailleurs indépendants travaillant pour leur propre compte et qui produisent des biens destinés exclusivement à une utilisation finale dans leur foyer.

01 Sur l'origine du terme de secteur informel, voir V. Tokman, Informality: exclusion and precariousness, article préparé pour le Colloque interrégional tripartite sur l'économie informelle : Permettre une transition vers la formalisation, OIT, Genève, 27-29 novembre 2007, p. 2, Voir également D. Kucera et L. Roncolato, « L'emploi informel : deux questions de politique controversées », Revue internationale du travail, vol. 147, $n^{\circ}$ 4, $2008, \mathrm{pp}$. 347-377 ; T. Packard, « Do workers in Chile choose informal employment ? A dynamic analysis of sector choice », Policy research working paper, $n^{\circ}$ 4232, Social Protection Unit, Banque Mondiale, mai 2007 ; J. Saavedra et M. Tommasi, «Informalité, État et contrat social en Amérique latine : étude préliminaire », Revue internationale du travail, 2007, Vol. 146, n³-4, p. 301 ; V. Tokman, «Informality: exclusion and precariousness », op. cit. ; V. Tokman, «Économie informelle, insécurité et cohésion sociale en Amérique latine », Revue internationale du travail, Vol. 146, n 1-2, 2007, p. 89 ; J.-M. Servais, « Secteur informel : un avenir pour le droit du travail », Actualités du droit, 1994, pp. 661-685.

02 OIT, Rapport de la Commission de l'économie informelle, Conférence internationale du Travail, 90e session, Genève, 2002, p. 58. Voir également BIT, Rapport VI, Travail décent et économie informelle, Conférence internationale du Travail, 90 session, Genève, 2002 ; BIT, L'économie informelle : permettre une transition vers la formalisation, Document du travail, Colloque interrégional tripartite sur l'économie informelle: Permettre une transition vers la formalisation, OIT, Genève, 27-29 novembre 2007

03 OIT, Emploi, revenus et égalité, stratégie pour accroître l'emploi productif au Kenya, Genève, OIT, 1972.

04 OIT, “Directrices sobre una definición estadística de empleo informal, adoptadas por la Decimoséptima Conferencia Internacional de Estadísticos del Trabajo (novembre - décembre 2003)". 
La doctrine s'est également efforcée de préciser le contenu de cette catégorie de travailleurs. Pour le professeur Marius Olivier ${ }^{05}$, les relations d'emploi informelles englobent les activités qui tombent, de facto ou de jure, en dehors de la portée de la loi, y compris de la sécurité sociale. L'économie informelle n'est pas une condition individuelle, mais un processus générant des revenus, qui se distingue par une caractéristique principale : il n'est pas réglementé par les institutions de la société, au sein d'un environnement juridique et social qui permet à des activités similaires d'être formellement encadrées. D'autre part, selon Victor Tokman ${ }^{06}$, deux facteurs ont probablement contribué à augmenter l'intérêt suscité par l'informalité. Le premier serait le lien étroit entre l'informalité, la pauvreté et le sous-emploi, avec une forte absorption des groupes les plus vulnérables de la société, y compris les femmes et les jeunes. Le second est la capacité d'adaptation du concept aux changements économiques et sociaux dans le monde.

Par conséquent, compte tenu du fait qu'il s'agit d'une catégorie complexe, aux contours multiples, et au regard de ses conséquences par rapport au statut des travailleurs concernés, on peut distinguer deux grandes formes d'unités productives ${ }^{07}$ :

- Les entreprises familiales, composées d'un travailleur indépendant (qui donne du travail), et de personnes qui collaborent avec lui (parfois ponctuellement), en l'absence d'existence légale de relations professionnelles subordonnées, mais de fait, en contrepartie d'une rémunération fixe, normale et habituelle ;

- Les microentreprises, ayant une taille et un degré d'organisation similaires aux précédentes mais avec la différence fondamentale que le titulaire procure des emplois salariés à des tiers, de façon continue.

En résumé, l'informalité du travail peut concerner aussi bien les travailleurs indépendants que les travailleurs subordonnés. La caractéristique commune est la précarité dans laquelle ils se trouvent et leur manque de protection, aussi bien en matière de droit du travail que de sécurité sociale ${ }^{08}$. Dans ce dernier cas, il convient de signaler que, si le système de sécurité sociale est basé sur des cotisations, cela entraîne des lacunes dans la couverture ${ }^{09}$, puisque tous les membres de ce marché ne pourront pas cotiser. Il faut tenir compte également du fait qu'il existe une déréglementation croissante du travail. Un nombre important de travailleurs du secteur formel ont des contrats précaires ${ }^{10}$, avec tous les risques que cela entraîne.

05 M. Olivier, "Work at the margins of social security: Expanding the boundaries of social protection in the developing world", in W. Van Oorschot, H. Peeters et K. Boos (eds), Invisible Social Security Revisited, Essays in Honour of Jos Berghman, Lannoo Publishers, Lovaina, Belgique, 2014, p. 215.

06 V. Tokman, Informality: exclusion and precariousness, op. cit., p. 2.

07 C. Lacchini, et G. Zuccotti, I mportancia de la contributividad en el sistema de seguridad social. Elementos de análisis para la inclusión social en América Latina. Proyecto de seguridad social para organizaciones sindicales SSOS, Première édition, Espagne, Centre international de formation de l'OIT, 2009, p. 27.

08 Pour les besoins de cet article, nous considérons que la sécurité sociale est « l'ensemble intégré des principes et des normes destinés à offrir une protection à toute personne confrontée à la survenance de risques sociaux par des transferts sociaux, qui peuvent être en espèces ou en nature, de financements contributifs ou non contributifs, dont l'administration peut être publique et/ou privée ; l'État devant agir en tant que garant de la protection de la population qui se trouve sur son territoire », in P. Arellano Ortiz, Lecciones de seguridad social, Librotecnia, 2017, pp.75-76.

09 F. Bertranou, [ed.], Envejecimiento, empleo y protección social en América Latina, Santiago, Organisation internationale du travail, 2006.

10 Ibid., p. 28. 
En général, en Amérique latine, les mécanismes de sécurité sociale ont été fortement influencés par le modèle bismarckien. Une grande partie de la population couverte bénéficie de cette couverture grâce à sa participation au marché du travail formel, à travers lequel elle contribue à la sécurité sociale. Cependant, la structure du marché du travail en Amérique du Sud nécessite un réexamen du financement traditionnel de la sécurité sociale dans le but d'élargir la couverture afin de protéger les personnes marginalisées. Il est également nécessaire que l'État augmente sa participation dans ce sens ${ }^{11}$. Les progrès sur la couverture sociale dans la région ont été apportés principalement à travers des mécanismes de transferts financiers conditionnels. Ces programmes ont eu une influence importante sur les progrès de la couverture de sécurité sociale dans la région américaine. À leur tour, ils ont eu un impact sur le marché du travail, ce qui implique un lien avec la capacité de contribuer aux systèmes de sécurité sociale.

Il a été signalé, dans un travail conjoint réalisé par la Comisión Económica para América Latina y el Caribe (CEPAL) des Nations Unies et de l'OIT'12, que les politiques publiques mettent l'accent sur les transferts financiers accordés dans le cadre de ces programmes. Cependant, en raison des montants réduits des transferts, ceux-ci n'ont généralement pas pour effet de diminuer l'insertion professionnelle des adultes en état de travailler mais plutôt, comme tel est leur objectif, de diminuer le travail des enfants. L'insertion professionnelle des adultes comme des jeunes - une fois leurs études terminées- est confrontée à la pénurie d'emplois productifs qui est, en général, particulièrement grave dans les zones connaissant des taux de pauvreté importants. Il faut rapprocher cela de l'insuffisance des mesures publiques en matière d'aides octroyées pour les soins aux enfants, aux personnes âgées et aux personnes handicapées, ce qui a un impact négatif sur la présence, sur le marché du travail, des femmes vivant dans des milieux défavorisés. Il est donc important que les programmes de transfert soient étroitement liés à des systèmes de protection sociale complets et à des politiques actives sur le marché du travail qui, entre autres objectifs, favorisent la demande de main-d’œuvre.

D'autre part, l'une des conclusions du travail conjoint est que la conception de ces programmes doit prendre en compte l'idée que les destinataires vont avoir à franchir plusieurs étapes, qui nécessitent un ensemble d'interventions, de sorte que le fait de «s'engager » dans les programmes ne puisse entraîner de rupture, comme la perte de leurs droits, ce qui pourrait décourager à terme ce processus de formalisation du travail. Par conséquent, la relation de ces programmes avec l'informalité est étroite, puisqu'elle stimule la formalisation des personnes qui en bénéficieront. Cependant, comme nous l'avons signalé, cette étude conclut que de tels programmes ont des effets limités.

Malgré les efforts déployés par les politiques publiques pour couvrir la population informelle plus largement en Amérique latine, le bilan fait état de succès et d'échecs. Sans aucun doute, le plus important est l'effort déployé pour inciter ces groupes à opter pour un travail formel, situation qui n'est pas toujours souhaitée. Le ratio moyen du travail informel dans la région est d'environ $50 \%$ de la population économiquement active. Dans certains pays, ce chiffre atteint même les $60 \%$. Même dans les pays où le taux est inférieur à $50 \%$, l'emploi informel est une dimension importante du marché du travail et a un impact significatif sur les mesures prises pour protéger cette catégorie de travailleurs ainsi que leurs familles ${ }^{13}$.

11 C. Lacchini et G. Zuccotti, Importancia de la contributividad en el sistema de seguridad social. Elementos de análisis para la inclusión social en América Latina. Proyecto de seguridad social para organizaciones sindicales SSOS, op. cit.

12 CEPAL/OIT, Los programas de transferencias condicionadas y el mercado laboral, Conyuntura laboral en América Latina y el Caribe, $n^{\circ} 10$, mai 2014, Nations Unies, Organisation Internationale du Travail, Santiago de Chile, 2014, p. 6.

13 OIT, Panorama Laboral 2016, Lima: OIT / Oficina Regional para América Latina y el Caribe, 2016, p. 38. 
Les mesures visant à protéger les travailleurs informels sont donc très variées. On peut, par exemple, citer : les mesures qui impliquent l'action des syndicats ${ }^{14}$; celles qui relèvent des lois sur l'immigration ${ }^{15}$; celles qui sont mises en œuvre à travers des mécanismes de protection sociale ${ }^{16}$, par exemple, les transferts financiers conditionnels ${ }^{17}$; et enfin celles qui sont mises en place grâce à des ajustements ou à l'adoption de normes du droit du travail.

Comme on peut le constater, aussi bien d'un point de vue doctrinal que pratique, la situation du travail informel dans la région latino-américaine se révèle complexe, tout en étant extrêmement importante. Chaque sous-région présente ses propres particularités et une analyse régionale s'avère être une tâche difficile. Aussi, nous nous concentrerons, dans cette contribution, sur la présentation succincte des principales mesures qui ont pu être adoptées afin de lutter contre l'informalité au Chili.

\section{I - Les mesures de protection du travail informel au Chili}

Le contexte du Chili est très particulier, car il est généralement présenté comme un pays ayant des résultats positifs dans le domaine économique, ce qui ne se vérifie pas nécessairement dans le domaine social. Si l'on situe le Chili dans le contexte de l'OCDE, ce pays est, aux côtés du Mexique, un des pays connaissant les plus grandes inégalités au sein de l'Organisation ${ }^{18}$. Mais d'un autre côté, le Chili a été donné en exemple pour ses politiques de protection des groupes exclus des mesures de protection par l'OIT ${ }^{19}$. Dans ce contexte, la politique appliquée dans les années 2000, envisageant la mise en place d'un programme nommé Chile Solidario, constitué par un mécanisme de protection sociale avec des transferts financiers conditionnels destinés aux familles les plus défavorisées pour une durée déterminée, a été très importante. Ce type de programme n'est pas exclusif au Chili puisqu'il existe aussi au Brésil, avec le programme Bolsa Familia, et au Mexique, avec le programme Oportunidades. Cependant, nous aimerions

14 Pour un exemple, voir P. Arellano Ortiz, Identificación de acciones y estrategias para mejorar la protección de las trabajadoras y trabajadores migrantes y sus familias mediante la seguridad social, Políticas de Migración Laboral Sensibles al Género en los Corredores de Nicaragua-Costa Rica-Panamá y HaitíRepública Dominicana, Organisation Internationale du Travail, Bureau de San José de Costa Rica, 2014.

15 Ibidem.

16 Aux fins de cet article, nous entendrons par « protection sociale » : l'ensemble des transferts de revenus (ou prestations), en espèces et en nature, qu'une société offre à ses membres individuellement, afin d'éviter ou d'alléger la pauvreté, ou d'aider à faire face à une série d'éventualités ou de risques de la vie qui, s'ils se produisaient, pourraient entraîner une perte de revenus. La perte de revenus peut résulter de la perte d'un emploi ou de l'incapacité à gagner de l'argent en raison de causes telles que l'invalidité ou la vieillesse, ou même de la nécessité de se procurer des soins médicaux coûteux en cas de maladie ou de détérioration de la santé, ou de diminuer ou corriger les inégalités créées à partir de la distribution primaire (avant les subventions) du revenu », M. Cichon, W. Scholz, Wolfgang, A. Van de Meerendonk, K. Hagemejer, F. Bertranou, et P. Plamondon, Financiación de la protección social, colección informes OIT, Ministerio de Trabajo y Asuntos Sociales, Madrid, 2006, p. 40.

17 Voir ECLAC/ ILO (2014) Los programas de transferencias condicionadas y el mercado laboral, Conyuntura laboral en América Latina y el Caribe, op. cit.

18 OCDE, Mise à jour sur les inégalités 2016 "Income inequality remains high in the face of weak recovery", Centre for Opportunity and Equality, 24 novembre 2016.

19 À ce sujet, consulter par exemple le rapport de l'OIT, Étendre la protection sociale à tous, un guide des défis et des options, Organisation internationale du travail, Département de la sécurité sociale, Genève, 2010 ; OIT OMS, Seuil de protection sociale pour une mondialisation juste et inclusive, Rapport du groupe consultatif sur la protection sociale (Genève, Organisation internationale du travail 2011) ; ILO, Extending Social Security to All, A Guide Through Challenges and Options (Genève, Organisation internationale du travail, Département de la sécurité sociale 2010). 


\section{L'INFORMALITÉ EN AMÉRIQUE LATINE}

avant tout souligner que ce type de mécanismes a produit des effets non seulement sur les travailleurs informels, mais aussi sur les couches les plus défavorisées de la population chilienne.

En ce qui concerne la mesure de l'informalité au Chili, on a toujours soutenu ${ }^{20}$ que le taux d'informalité dans ce pays restait inférieur aux niveaux observés dans la région latino-américaine. Si l'on prend la période 2010-2014, à partir des données de l'étude NENE INueva encuesta nacional de empleo ${ }^{21}$ ), il s'est produit une diminution continue du taux d'informalité jusqu'à la fin de l'année 2012, de $39,4 \%$ à $35,7 \%$, alors qu'en 2014 , une légère augmentation pendant la première moitié de l'année a pu être constatée.

Afin d'analyser les mesures établies au Chili, nous nous intéresserons d'abord à leur institutionnalisation (A), puis au système de sécurité et d'opportunité (SeguridadesyOportunidades), qui est le successeur de Chile Solidario (B) et, enfin, nous ferons référence à une série d'avantages et de subventions destinés aux travailleurs informels dans diverses situations (C).

\section{A - L'institutionnalisation de la protection}

Il est pertinent de s'intéresser à cette institutionnalisation car on peut noter qu'une similitude existe avec d'autres pays dans la manière de traiter la question de l'informalité et de sa protection ${ }^{22}$. Ainsi, ces questions ont été traitées par le ministère généralement dit du Développement social.

Les origines du programme Seguridades y Oportunidadesse trouvent dans l'activité du ministère de la planification. En 2002, le Président de la République du Chili, Ricardo Lagos, a présenté un programme innovant baptisé Chile Solidario. L'objectif de ce programme était d'intégrer les familles vivant dans une pauvreté extrême dans le réseau public de protection sociale, de manière garantie et/ou préférentielle. Dans le cadre de ce programme, la famille était considérée comme le nouveau centre d'intervention sociale. Ce choix a été fait sur la base d'informations qui ont démontré une plus grande efficacité des programmes sociaux lorsqu'ils sont axés sur la famille et les femmes au sein de la famille, en tant que principales victimes de la pauvreté. En outre, le programme a souligné la nécessité de fournir des services psychologiques et sociaux aux familles vivant dans une extrême pauvreté.

En résumé, ce soutien a permis aux familles d'obtenir des résultats concrets et de maintenir ces résultats dans le temps, améliorant ainsi leur qualité de vie et pas seulement leurs revenus.

Le système Chile Solidario comprenait trois composantes principales pour aider la population cible :

- un soutien psychosocial et des bons de protection familiale ;

- des subventions financières garanties;

- l'accès préférentiel aux programmes de développement social.

20 CIEDESS, Análisis y propuestas para incentivar la cotización de los trabajadores al sistema de pensiones chileno, informe final (Santiago, 29 janvier 2015).

21 Nouvelle enquête nationale sur l'emploi. Cette enquette peut être consultée sur le site http://www.ine.cl

22 À ce sujet, voir T. Dijkhoff et L. George Mpedi (éd.), Recommendation on Social Protection Floors. Basic Principles for Innovative Solutions, Studies in Employment and Social Policy, Kluwer Law International, 2017. 
Cependant, même si l'on considère que le programme a été un succès dans la lutte contre la pauvreté, rien ne prouve de manière significative qu'il ait contribué à augmenter le niveau moyen d'emploi ou de rémunération des participants. Il faut souligner que les évaluations menées ont une portée très limitée, en raison du peu d'informations disponibles ${ }^{23}$. Ainsi, le programme a aidé les pauvres à survivre mais n'a pas franchi l'étape suivante, consistant à les faire sortir de la pauvreté.

Les origines du Ministère de la Planification remontent à l'époque du Gouvernement du Président Eduardo Frei Montalva (1964-1970), lorsque le besoin s'est fait sentir de disposer d'une organisation technique dédiée à la planification du développement à l'échelle nationale. Le bureau de la planification (Oficina de Planificación ou ODEPLAN) a donc été créé et, au cours des deux premières années, il a fonctionné sans reconnaissance légale et a rendu compte directement au Président de la République. En 1967, cette nouvelle entité a acquis le statut d'un service public décentralisé, doté de fonds propres. Les bureaux régionaux de planification (Oficinas Regionales de Planificación), qui ont par la suite été rebaptisés Secrétariats régionaux de planification et de coordination (Secretarías Regionales de Planificación y Coordinación ou SERPLAC), ont joué le rôle d'organes consultatifs, et ont créé le Secrétariat technique du gouverneur régional et le Conseil de développement régional.

En 1990, par le décret-loi 18.899 du 19 juillet 1990, le Bureau national de planification a été transformé en Ministère de la planification et de la coopération, chargé de collaborer avec le Président de la République pour la conception et la mise en œuvre des plans et des programmes nationaux de développement, de proposer des objectifs d'investissement public, d'évaluer les projets d'investissement financés par l'État et de coordonner les diverses initiatives du secteur public visant à éradiquer la pauvreté.

Cet effort visant à optimiser les politiques publiques au bénéfice des habitants les plus démunis du pays a motivé la création, en 2002, du système Chile Solidario, qui apportait un soutien complet aux personnes et aux familles vivant dans une extrême pauvreté. Trois ans plus tard, le secrétaire d'État a été chargé de développer, de coordonner, de diffuser et de promouvoir au niveau national le système de protection sociale, qui a radicalement modifié l'approche des politiques sociales, laissant derrière lui une vision paternaliste et la remplaçant par une focalisation sur les droits individuels.

En octobre 2011, Mideplan est devenu le Ministère du Développement social, élargissant ses pouvoirs et se positionnant comme l'organe de coordination de toutes les politiques sociales chiliennes (politiques de protection sociale), coordonnant les initiatives interministérielles et supervisant leur fonctionnement.

La création de deux nouveaux sous-secrétariats est l'une des grandes innovations de ce portefeuille. Le sous-secrétariat aux services sociaux est chargé de coordonner les activités de tous les services connexes : le Service national pour les personnes âgées (Senama), la Société nationale de développement indigène (Conadi), le Fonds de solidarité et d'investissement social (Fosis) l'Institut de la Jeunesse (Injuv) et le Service national pour les personnes handicapées (Senadis). Il coordonne également l'ensemble du système de protection sociale, en lien avec d'autres ministères.

23 0. Larrañaga, D. Contreras et G. Cabezas, Políticas Contra la Pobreza: de Chile Solidario al Ingreso Ético Familiar, Documento de Trabajo, Programme des Nations unies pour le Développement, 29 (Chile, Área de Reducción de la Pobreza y la Desigualdad, Décembre 2014). 
Le sous-secrétariat à l'évaluation sociale a également été créé, afin d'améliorer l'impact des politiques sociales, d'évaluer et de contrôler les programmes sociaux existants, afin de s'assurer que les fonds publics sont dépensés de manière adéquate et parviennent aux bénéficiaires visés. Ainsi, le Ministère du Développement social a emprunté une nouvelle voie, en mettant l'accent sur la lutte contre la pauvreté et les besoins des membres les plus vulnérables et discriminés de la société.

Le sous-secteur Seguridades y Oportunidades, créé en 2012 en vertu de la loi 20.59524, promeut le travail réalisé par Chile Solidario concernant la population extrêmement défavorisée et les groupes vulnérables déjà évoqués. Dans ce contexte, les personnes et les familles qui ont fait partie du programme Chile Solidario à la fin de l'année 2012 resteront dans le programme jusqu'à la fin de leur participation. À l'heure actuelle, ces personnes et ces familles ne peuvent rejoindre le programme Seguridades y Oportunidades qu'à la fin du programme Chile Solidario, ce qui laisse un groupe de 50683 familles bénéficiaires actives et, pour la plupart d'entre elles, cette participation prendra fin en $2017^{25}$.

Le nombre total d'utilisateurs au 31 décembre 2014 correspondait à 106990 foyers, ce qui représente 399588 participants. Sur ce total, 55,7\% étaient des femmes et les $44,3 \%$ restants étaient des hommes ${ }^{26}$.

Traditionnellement, au Chili, la pauvreté se mesure sur la base du revenu familial. Ce système de mesure de la pauvreté monétaire constitue une mesure indirecte du bienêtre accessible aux personnes et à leurs foyers, à travers la consommation de biens et de services. Cette approche vise à simplifier l'identification de ceux qui sont considérés comme vivant selon un niveau de vie acceptable, en tenant compte d'un seul indicateur. Pourtant, une mesure multidimensionnelle de la pauvreté est préférable, car elle permet de mesurer directement les conditions de vie de la population, en regardant la situation des personnes et des ménages sous différents aspects et sur la base d'indicateurs de bien-être considérés comme socialement pertinents. De cette manière, il est possible d'identifier l'ensemble des déficiences qui affectent les personnes et leurs foyers, au-delà de la seule question du revenu, et d'analyser l'importance relative de ces déficiences dans les conditions de vie de la population vivant dans la pauvreté2 ${ }^{27}$. C'est cette redéfinition qui a rendu le Chili capable de mieux cerner la situation des pauvres et de pouvoir ainsi mieux les protéger.

Le Registre social des foyers (RSH) est le nouveau système de soutien à la sélection des bénéficiaires des prestations sociales, qui, le $1^{\text {er }}$ janvier 2016, a remplacé le fichier de protection sociale (FPS). L'objectif est de soutenir les différents processus de sélection des bénéficiaires des avantages et des programmes sociaux, et de fournir un ensemble complet d'informations, principalement à partir des dossiers administratifs de l'État, ce qui permet la construction d'une classification socioéconomique des foyers ${ }^{28}$.

24 Cette loi s'appelle Ley que Crea el Ingreso ético familiar (loi établissant le revenu d'éthique familiale) qui fixe des primes et des subventions conditionnées pour les familles vivant dans une extrême pauvreté et crée des subventions pour l'emploi des femmes, publiée au Journal officiel le 17 mai 2012.

25 Ministère du développement social, Informe de Desarrollo Social 2014 (Santiago de Chile, 2014).

26 Ministère du développement social, Informe de Desarrollo Social 2015, 112 (Santiago de Chile, 2015).

27 Ministère du développement social, Nueva Metodología de Medición de la Pobreza por Ingresos y Multidimensional, Serie Documentos Metodológicos n² 28, 24 (Santiago de Chile, 2015).

28 Ministère du développement social, Nueva Metodología de Medición de la Pobreza por Ingresos y Multidimensional, Serie Documentos Metodológicos n²8, 24 (Santiago de Chile, 2015). 


\section{B - Sécurité et opportunités dans le cadre des prestations de protection sociale}

Depuis le renouvellement du programme, les sous-programmes ont également été reformulés. Les programmes dirigés aujourd'hui par le ministère du Développement social sont les suivants:

(1) Programme Chile Crece Contigo/Crece con usted (le Chili grandit avec toi/avec vous)

Chile Crece con Usted a été créé en 2007, afin d'accompagner, de protéger et de soutenir les enfants dès la grossesse et pendant les premières années de leur vie. C'est un système complet pour les enfants, avec des activités et des services fournis par différents départements gouvernementaux coordonnés par le Ministère de l'Éducation.

Il fait partie du Système intersectoriel de protection sociale (loi 20.379), qui regroupe des actions et des avantages sociaux pour les personnes vivant dans des situations les plus extrêmes de vulnérabilité socioéconomique.

Les enfants sont accueillis pour la première fois dans le cadre du programme Chile Crece Contigo dès le premier rendez-vous prénatal prévu dans le cadre du système de santé publique. Ils sont ensuite accompagnés et soutenus pendant leur développement jusqu'à leur entrée dans le système scolaire. Ils ont également un accès garanti aux aides techniques, crèches, garderies, et jardins d'enfants.

\section{(2) Programme Seguridades y Oportunidades}

Afin de remédier aux inégalités et d'ouvrir plus de possibilités aux femmes et aux hommes de différents âges et conditions, le Ministère du Développement social a créé des programmes, des actions et des avantages sociaux qui constituent le système de protection sociale.

Les bénéficiaires qui participent à ces programmes ont accès, en première instance, à une assistance psychosociale consistant en un soutien permanent dispensé par un professionnel qui travaille avec la famille et/ou la personne pendant le processus. En outre, les participants ont accès à une aide à l'emploi, également avec le soutien d'un professionnel, au renforcement des capacités et peuvent améliorer leurs chances de trouver du travail et un emploi.

Les participants ont un accès garanti à une contribution économique, sur présentation d'un coupon, ainsi qu'à une aide monétaire pour compléter le revenu déjà perçu par la personne et/ou la famille. L'accès aux services sociaux et aux avantages sociaux est également garanti pour soutenir les personnes en fonction de leur statut et de leurs besoins spécifiques. 
(3) Programme familial

Le programme familial s'adresse aux individus et aux familles vivant dans une extrême pauvreté et particulièrement vulnérables. Il comprend un ensemble d'actions, de services sociaux et d'avantages pour améliorer la qualité de vie des individus et des familles, ce qui contribue à l'obtention d'un degré plus élevé de bien-être et de cohésion sociale.

Pour remplir cet objectif, le programme propose un soutien psychosocial et en matière de gestion, des services sociaux et des avantages, ainsi que des primes et des subventions. Le Fonds de solidarité et d'investissement social (FOSIS) assure la mise en œuvre de ce programme au niveau local par les municipalités et les régions.

\section{(4) Programme Abriendo Caminos}

Le programme Abriendo Caminos vise à prendre des mesures préventives et correctives dans les conditions de développement des enfants et des adolescents privés de liberté.

À cette fin, il favorise le développement des capacités et des compétences qui leur permettent d'obtenir de meilleures conditions de vie, grâce à une stratégie d'intervention globale et personnalisée. Les différentes actions et le soutien accordé sont définis en fonction des particularités, des besoins, des ressources et des capacités de chacun des membres de la famille. Le programme a été lancé en 2008 et, depuis 2012, a été mis en œuvre au niveau national.

\section{(5) Programme Vinculos}

Le programme Vinculos offre un soutien complet aux adultes et personnes âgées de plus de 65 ans, vivant dans les conditions de vulnérabilité et d'exclusion sociale. Il se compose d'un ensemble d'actions ciblées pour répondre directement aux besoins des personnes appartenant à des ménages d'une ou deux personnes. En outre, il crée les conditions d'accès au réseau communautaire de protection sociale, en reliant les personnes âgées aux services publics et aux réseaux sociaux de leur communauté. Le programme Vinculos offre à ses participants un emploi et un soutien psychosocial.

\section{C - Les primes d'aide aux travailleurs informels}

La protection du travailleur informel ne se concentre pas uniquement sur les mécanismes de protection sociale. D'autres types de mesures ont également été adoptés, qui sont difficiles à résumer brièvement. Il s'agit de primes spécifiques composées d'un versement unique ou de versements périodiques, limités dans le temps, et dont les bénéficiaires potentiels sont également en nombre limité. Ces prestations sociales ont un impact particulier sur les groupes exclus, tels que les travailleurs informels.

Parmi ces mesures, nous souhaitons mentionner la prime de formalisation, ainsi que la subvention pour l'emploi des femmes, ces deux avantages figurant dans la loi n 20.595 déjà mentionnée. Il s'agit dans les deux cas de prestations sociales non contributives financées par le budget de l'État. Ce ne sont pas des programmes d'insertion sur le marché du travail. De tels programmes existent au Chili, mais avec une approche plus générale et sans incitation financière. Ces deux prestations sociales nous intéressent donc car l'une est une récompense accordée aux personnes qui passent à la formalité et l'autre a pour but de protéger les femmes qui travaillent. 


\section{Prime de formalisation}

Elle figure à l'article 19 de la loi $n^{0} 20.595$, qui prévoit une prime pour les réalisations du sous-système. Elle est destinée aux personnes et/ou aux familles qui utilisent le sous-système de Seguridades y Oportunidades et qui participent au programme d'accompagnement socioprofessionnel. Elles auront droit à la prime de formalisation, à condition que pendant leur participation à ce programme, leurs cotisations à la sécurité sociale soient déclarées et payées continuellement pour une période minimale déterminée de manière réglementaire ${ }^{29}$. La prime versée pour la formalisation consiste en un paiement de $200000 \$^{30}$, versé une seule fois selon un quota, réajusté en février de chaque année, en fonction de la variation de l'Indice des prix à la consommation (IPC).

\section{Subvention pour l'emploi des femmes}

Cet avantage est défini dans l'article 21 de la loi $n^{0} 20.595$, qui établit une subvention pour l'emploi des travailleuses subordonnées relevant du Code du travail et des travailleuses indépendantes, ce qui représente une charge fiscale. Les travailleuses subordonnées et indépendantes, âgées de 25 à 60 ans et qui font partie des $40 \%$ de personnes les plus vulnérables sur le plan socioéconomique, ainsi que leurs employeurs respectifs, ont droit à cette prime à l'emploi.

Cette subvention peut être perçue par chaque travailleuse pendant quatre années consécutives. Une même travailleuse fera bénéficier, au maximum pendant vingt-quatre mois, de subventions pour le ou les employeurs pour lesquels elle travaille. Pour l'année 2016, on estime qu'il y a eu un total de 2000 bénéficiaires ${ }^{31}$.

\section{Conclusion}

Comme nous l'avons souligné ${ }^{32}$, l'évolution décrite montre que le Chile Solidarioconstitue sans aucun doute un excellent exemple de la prise de conscience d'un gouvernement de la nécessité de reformuler les politiques publiques. L'approche pluridisciplinaire actuelle est sans aucun doute compatible avec l'idée d'une meilleure protection des groupes vulnérables, au sein desquels les travailleurs informels les plus nécessiteux sont les bénéficiaires de ces aides.

Cependant, nous avons également indiqué que le niveau des prestations fournies se situait juste au-dessus du seuil de pauvreté. Par conséquent, afin d'apporter un changement significatif dans ce groupe, le montant des subventions octroyées devrait augmenter de façon substantielle, faute de quoi ce dispositif aidera uniquement les pauvres à rester pauvres, au lieu de les aider à s'extirper du piège de la pauvreté.

29 Actuellement, on exige quatre cotisations mensuelles continues pour les régimes de santé, de retraite ou d'assurance chômage, déclarées et payées pendant la période de participation effective à l'accompagnement socioprofessionnel.

30 Équivalant à 267 euros.

31 Ministère du développement social, Informe de Desarrollo Social 2016, p. 111, Santiago de Chile, 2016.

32 P. Arellano Ortiz, "Chile Solidario CCT Programme: New Logic Behind Chilean Social Protection Programmes", T. Dijkhoff et L. George Mpedi (éditeurs), Recommendation on Social Protection Floors. Basic Principles for Innovative Solutions, Studies in Employment and Social Policy, Kluwer Law International, 2017, pp. 197-218. 


\section{L'INFORMALITÉ EN AMÉRIQUE LATINE}

Ces mécanismes aident donc souvent les travailleurs informels à survivre, mais pas à échapper à leur situation précaire. L'exclusion est maintenue dans une certaine mesure par la loi. Dans une telle situation, il convient de rechercher un mécanisme de protection spécialement destiné aux travailleurs informels. Il ne s'agit pas de forcer les travailleurs informels à passer à la formalité, ce que fait par exemple la recommandation $n^{\circ} 204$ de l'OIT ${ }^{33}$, et à obtenir ainsi une protection traditionnelle. Il s'agit plutôt de comprendre les travailleurs informels et leurs besoins, de les protéger sans leur imposer des modèles de travail qu'ils ne connaissent pas et dont ils ne souhaitent pas faire partie ${ }^{34}$. Les défis, pour la législation sociale, sont importants et exigent une reformulation de politiques afin de permettre l'incorporation de tous les exclus, dont les travailleurs informels font partie, mais sans affecter les mécanismes qui leur permettent de subvenir à leurs besoins.

33 Recommandation $n^{\circ} 204$ sur la transition de l'économie informelle à l'économie formelle, adoptée lors de la $104^{\mathrm{e}}$ Conférence Internationale du Travail le 12 juin 2015 à Genève.

34 Nous le soulignons dans P. Arellano Ortiz, Seguridad sociale: cómo llegar al 2050 con un sistema sano económica y socialmente, commentaires sur la présentation principale du $10^{\text {e }}$ Congrès régional américain sur le droit du travail et la sécurité sociale, qui s'est tenu à Panama, République de Panama, du 26 au 30 septembre 2016, organisé par la Société internationale du droit du travail (SIDTSS) et l'Académie panaméenne du droit du travail (APADETRA).

\section{PABLO ARELLANO ORTIZ}

Professeur de Droit du Travail et de Sécurité Sociale, Pontificia Universidad Católica de Valparaíso.

Thèmes de recherche : État Providence, retraite, accidents du travail, normes internationales du travail et protection des travailleurs de l'économie informelle.

Parmi ses publications :

P. Arellano Ortiz, Lecciones de seguridad social, 2da Edición, Librotecnia, Santiago, junio 2017.

P. Arellano Ortiz, S. Gamonal Contreras, «Flexibilidad y desigualdad en Chile: el Derecho Social en un contexto neoliberal », Boletín Mexicano de Derecho Comparado, nueva serie, año XLX, núm. 149, mayo-agosto de 2017, pp. 555-579. 